\title{
Front Matter: Volume 9136
}

, "Front Matter: Volume 9136," Proc. SPIE 9136, Nonlinear Optics and Its Applications VIII; and Quantum Optics III, 913601 (20 May 2014); doi: 10.1117/12.2069524

SPIE. Event: SPIE Photonics Europe, 2014, Brussels, Belgium 


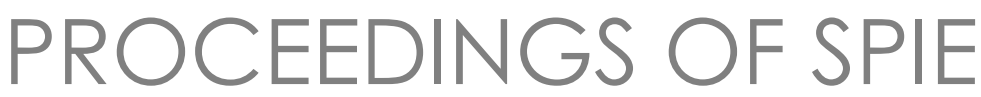

\title{
Nonlinear Optics and Its Applications VIII; and Quantum Optics III
}

\author{
Benjamin J. Eggleton \\ Alexander L. Gaeta \\ Neil G. R. Broderick \\ Alexander V. Sergienko \\ Arno Rauschenbeutel \\ Thomas Durt \\ Editors
}

\section{4-16 April 2014 \\ Brussels, Belgium}

Sponsored by

SPIE

Cosponsored by

B-PHOT_Brussels Photonics Team (Belgium) - FWO_Fonds Wetenschappelijk Onderzoek (Belgium) • Brussels-Capital Region (Belgium) • Ville de Bruxelles (Belgium)

Cooperating Organisations

CBO-BCO (Belgium) • European Laser Institute • Photonics 21 (Germany) • EOS—European Optical Society (Germany)

Published by

SPIE 
The papers included in this volume were part of the technical conference cited on the cover and title page. Papers were selected and subject to review by the editors and conference program committee. Some conference presentations may not be available for publication. The papers published in these proceedings reflect the work and thoughts of the authors and are published herein as submitted. The publisher is not responsible for the validity of the information or for any outcomes resulting from reliance thereon.

Please use the following format to cite material from this book:

Author(s), "Title of Paper," in Nonlinear Optics and Its Applications VIII; and Quantum Optics III, edited by Benjamin J. Eggleton, Alexander L. Gaeta, Neil G. R. Broderick, Alexander V. Sergienko, Arno Rauschenbeutel, Thomas Durt, Proceedings of SPIE Vol. 9136 (SPIE, Bellingham, WA, 2014) Article CID Number.

ISSN: 0277-786X

ISBN: 9781628410846

Published by

SPIE

P.O. Box 10, Bellingham, Washington 98227-0010 USA

Telephone +1 3606763290 (Pacific Time) · Fax +1 3606471445

SPIE.org

Copyright (C) 2014, Society of Photo-Optical Instrumentation Engineers.

Copying of material in this book for internal or personal use, or for the internal or personal use of specific clients, beyond the fair use provisions granted by the U.S. Copyright Law is authorized by SPIE subject to payment of copying fees. The Transactional Reporting Service base fee for this volume is $\$ 18.00$ per article (or portion thereof), which should be paid directly to the Copyright Clearance Center (CCC), 222 Rosewood Drive, Danvers, MA 01923. Payment may also be made electronically through CCC Online at copyright.com. Other copying for republication, resale, advertising or promotion, or any form of systematic or multiple reproduction of any material in this book is prohibited except with permission in writing from the publisher. The CCC fee code is 0277-786X/14/\$18.00.

Printed in the United States of America.

Publication of record for individual papers is online in the SPIE Digital Library.

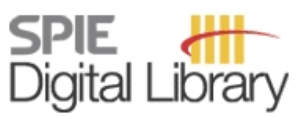

SPIEDigitallibrary.org

Paper Numbering: Proceedings of SPIE follow an e-First publication model, with papers published first online and then in print and on CD-ROM. Papers are published as they are submitted and meet publication criteria. A unique, consistent, permanent citation identifier (CID) number is assigned to each article at the time of the first publication. Utilization of CIDs allows articles to be fully citable as soon as they are published online, and connects the same identifier to all online, print, and electronic versions of the publication. SPIE uses a six-digit CID article numbering system in which:

- The first four digits correspond to the SPIE volume number.

- The last two digits indicate publication order within the volume using a Base 36 numbering

system employing both numerals and letters. These two-number sets start with 00, 01, 02, 03, 04, $05,06,07,08,09,0 A, 0 B \ldots$. 0Z, followed by 10-1Z, 20-2Z, etc.

The CID Number appears on each page of the manuscript. The complete citation is used on the first page, and an abbreviated version on subsequent pages. Numbers in the index correspond to the last two digits of the six-digit CID Number. 


\section{Contents}

xi Conference Committee

xv Quantum Optics Introduction

\section{Part A Nonlinear Optics and its Applications}

NONLINEAR QUANTUM OPTICS

913603 Domain-engineered PPLN for entangled photon generation and other quantum information applications (Invited Paper) [9136-2]

P. S. Kuo, National Institute of Standards and Technology (United States); J. S. Pelc, HewlettPackard Labs. (United States); O. Slattery, L. Ma, X. Tang, National Institute of Standards and Technology (United States)

913604 Efficient four-wave mixing by phase-mismatch switching (Best Student Paper Award) [9136-3]

Y. Lefevre, N. Vermeulen, H. Thienpont, Vrije Univ. Brussel (Belgium)

913606 Indirect transitions of a signal interacting with a moving refractive index front [9136-5]

M. Castellanos Muñoz, A. Yu. Petrov, Technische Univ. Hamburg-Harburg (Germany);

L. O'Faolain, Univ. of St. Andrews (United Kingdom); J. Li, Sun Yat-Sen Univ. (China);

T. F. Krauss, The Univ. of York (United Kingdom); M. Eich, Technische Univ. Hamburg-Harburg (Germany)

\section{HIGH POWER EFFECTS}

913608 Longitudinal mode-filling to cancel SBS in fully-fibered MOPAs dedicated to the production of high-energy nanosecond pulses [9136-7]

A. Jolly, ALPHANOV, Institut d'Optique d'Aquitaine (France) and CEA-CESTA (France);

F. S. Gokhan, Hasan Kalyoncu Univ. (Turkey); R. Bello, P. Dupriez, ALPHANOV, Institut

d'Optique d'Aquitaine (France)

913609 Laser-induced microwave generation with nonlinear optical crystals [9136-8]

F. A. Borghesani, Istituto Nazionale di Fisica Nucleare (Italy) and CNISM (Italy); C. Braggio, Univ. degli Studi di Padova (Italy) and Istituto Nazionale di Fisica Nucleare (Italy);

G. Carugno, Istituto Nazionale di Fisica Nucleare (Italy); F. Della Valle, Univ. degli Studi di Trieste (Italy); G. Ruoso, Istituto Nazionale di Fisica Nucleare (Italy) 
9136 OD High-yield second-harmonic generation from mid-infrared to near-infrared regions in silicon-organic hybrid plasmonic waveguides [9136-12]

J. Zhang, Huazhong Univ. of Science and Technology (China) and Institut d'Electronique Fondamentale, Univ. Paris-Sud, CNRS (France); X. Zhang, Huazhong Univ. of Science and Technology (China); E. Cassan, Institut d'Electronique Fondamentale, Univ. Paris-Sud, CNRS (France)

9136 OE All-optical generation of surface acoustic waves in a silica optical microwire [9136-13] J.-C. Beugnot, Institut FEMTO-ST, CNRS-Univ. de Franche-Comté (France); S. Lebrun, G. Pauliat, Lab. Charles Fabry, Institut d'Optique, Univ. Paris-Sud, CNRS (France); H. Maillotte, Institut FEMTO-ST, CNRS-Univ. de Franche-Comté (France); V. Laude, Lab. Charles Fabry, Institut d'Optique, Univ. Paris-Sud, CNRS (France); T. Sylvestre, Institut FEMTOST, CNRS-Univ. de Franche-Comté (France)

9136 OG Opportunities for Raman wavelength conversion with silicon microdisks [9136-15]

I. Degli-Eredi, N. Vermeulen, H. Thienpont, Vrije Univ. Brussel (Belgium)

\section{CAVITY SOLITONS}

9136 0J Spatio-temporal stability of ID Kerr cavity solitons [9136-18]

L. Gelens, Vrije Univ. Brussel (Belgium) and Stanford Univ. School of Medicine (United States); P. Parra-Rivas, Vrije Univ. Brussel (Belgium) and Instituto de Fisica Interdisciplinar y Sistemas Complejos (Spain); F. Leo, Univ. Gent (Belgium) and IMEC (Belgium); D. Gomila, M. A. Matias, Instituto de Fisica Interdisciplinar y Sistemas Complejos (Spain); S. Coen, The Univ. of Auckland (New Zealand)

9136 OK Strong nonlocal interaction stabilizes cavity solitons with a varying size plateau [9136-19] C. Fernandez-Oto, M. Tlidi, Univ. Libre de Bruxelles (Belgium); D. Escaff, Univ. de Los Andes (Chile); M. Clerc, Univ. de Chile (Chile); P. Kockaert, Univ. Libre de Bruxelles (Belgium)

\section{SPECIALTY FIBRES}

$9136 \mathrm{ON} \quad$ Controlling modulation instability using an incoherent low amplitude seed [9136-22] T. Godin, D. M. Nguyen, S. Toenger, Y. Combes, B. Wetzel, T. Sylvestre, Institut FEMTO-ST, CNRS-Univ. de Franche-Comté (France); G. Genty, Tampere Univ. of Technology (Finland); F. Dias, Univ. College Dublin (Ireland); J. M. Dudley, Institut FEMTO-ST, CNRS-Univ. de Franche-Comté (France)

913600 Tunable stimulated Brillouin scattering in hybrid polymer-chalcogenide tapered fibers [9136-23]

J.-C. Beugnot, Institut FEMTO-ST, CNRS, Univ. de Franche-Comté (France); R. Ahmad, M. Rochette, McGill Univ. (Canada); V. Laude, H. Maillotte, T. Sylvestre, Institut FEMTO-ST, CNRS, Univ. de Franche-Comté (France) 
9136 OP Normal dispersion modulation instability in an $\mathrm{As}_{2} \mathrm{Se}_{3}$ chalcogenide hybrid microwire [9136-24]

T. Godin, Y. Combes, Institut FEMTO-ST, CNRS, Univ. de Franche-Comté (France); R. Ahmad, M. Rochette, McGill Univ. (Canada); T. Sylvestre, J. M. Dudley, Institut FEMTO-ST, CNRS, Univ. de Franche-Comté (France)

$91360 Q$ Temporal localized structures in a photonic crystal fiber resonator [9136-25]

L. Bahloul, L. Cherbi, H. Hariz, Univ. des Sciences et de la Technologie Houari Boumediene (Algeria)

\section{APPLICATIONS OF NONLINEAR OPTICS}

9136 OS Airy beams propagation in optically induced photonic lattices [9136-27] B. Bokić, Univ. of Belgrade (Serbia); F. Diebel, Westfälische Wilhelms-Univ. Münster (Germany); D. Timotijević, A. Piper, Univ. of Belgrade (Serbia); M. Boguslawski, Westfälische Wilhelms-Univ. Münster (Germany); D. Jović, Univ. of Belgrade (Serbia); C. Denz, Westfälische Wilhelms-Univ. Münster (Germany)

9136 OV Parallel generation of fast random bits based on optoelectronic phase-chaos systems [9136-30]

R. M. Nguimdo, Vrije Univ. Brussel (Belgium); P. Colet, Consejo Superior de Investigaciones Científicas (Spain); J. Danckaert, Vrije Univ. Brussel (Belgium)

\section{FIBRE LASERS I}

9136 OW High-power femtosecond fiber lasers based on self-similar pulse evolution (Invited Paper) [9136-31]

H. Liu, W. Renninger, Cornell Univ. (United States); B. Nie, M. Dantus, Michigan State Univ. (United States); F. Yu, J. Knight, Univ. of Bath (United Kingdom); A. Chong, Univ. of Dayton (United States); F. Wise, Cornell Univ. (United States)

9136 OX Vector solitons in harmonic mode-locked erbium-doped fiber lasers [9136-32] T. Habruseva, Aston Univ. (United Kingdom); M. Mkhitaryan, Moscow Institute of Physics and Technology (Russian Federation); C. Mou, A. Rozhin, S. K. Turitsyn, S. V. Sergeyev, Aston Univ. (United Kingdom)

$9136 \mathrm{OZ}$ Self-similar pulse-shape mode for femtosecond pulse propagation in medium with resonant nonlinearity [9136-34]

V. A. Trofimov, I. G. Zakharova, Lomonosov Moscow State Univ. (Russian Federation);

S. Konar, Birla Institute of Technology (India) 
913610 Nonlinear energy deposition in water from fs-laser pulses: effect of the input chirp [9136-35] C. Milián, Ctr. de Physique Theorique, CNRS, Ecole Polytechnique (France); A. Jarnac, Y. Brelet, Lab. d'Optique Appliquee, ENSTA ParisTech, Ecole Polytechnique, CNRS (France); V. Jukna, Ctr. de Physique Theorique, CNRS, Ecole Polytechnique (France); A. Houard, A. Mysyrowicz, Lab. d'Optique Appliquee, ENSTA ParisTech, Ecole Polytechnique, CNRS (France); A. Couairon, Ctr. de Physique Theorique, CNRS, Ecole Polytechnique (France)

913612 Nonlinear mixing and mode correlations in a short Raman fiber laser [9136-37] I. D. Vatnik, Institute of Automation and Electrometry (Russian Federation); O. A. Gorbunov, Institute of Automation and Electrometry (Russian Federation) and Novosibirsk State Univ. (Russian Federation); D. V. Churkin, Institute of Automation and Electrometry (Russian Federation), Novosibirsk State Univ. (Russian Federation), and Aston Univ. (United Kingdom)

913613 Influence of the generated power, measurement bandwidth, and noise level on intensity statistics of a quasi-CW Raman fiber laser [9136-38]

O. A. Gorbunov, Institute of Automation and Electrometry (Russian Federation) and Novosibirsk State Univ. (Russian Federation); S. Sugavanam, Aston Univ. (United Kingdom); D. V. Churkin, Institute of Automation and Electrometry (Russian Federation), Novosibirsk State Univ. (Russian Federation), and Aston Univ. (United Kingdom)

\section{PARAMETRIC EFFECTS}

913614 Sensitive terahertz-wave detector using a quasi-phase-matched $\mathrm{LiNbO}_{3}$ at room temperature [9136-40]

K. Nawata, T. Notake, RIKEN Ctr. for Advanced Photonics (Japan); H. Ishizuki, Institute for Molecular Science (Japan); F. Qi, Y. Takida, S. Fan, S. Hayashi, RIKEN Ctr. for Advanced Photonics (Japan); T. Taira, Institute for Molecular Science (Japan); H. Minamide, RIKEN Ctr. for Advanced Photonics (Japan)

$9136162.6 \mu \mathrm{m}$ to $\mathbf{1 2} \boldsymbol{\mu \mathrm { m }}$ tunable ZGP parametric master oscillator power amplifier [9136-42] T. Traub, Photonik-Zentrum Kaiserslautern e.V. (Germany); G. Anstett, Fraunhofer-Institut für Optronik, Systemtechnik und Bildauswertung (Germany); G. Goeritz, GWU-Lasertechnik Vertriebsges.mbH (Germany); J. L'huillier, Photonik-Zentrum Kaiserslautern e.V. (Germany)

POSTER SESSION

913618 Field-enhanced nonlinear optical properties of organic nanofibers [9136-39] O. Kostiučenko, J. Fiutowski, J. R. Brewer, H.-G. Rubahn, Univ. of Southern Denmark (Denmark)

913619 Multi-modes of four-waves mixing at non-collinear interaction of laser beams in medium with cubic nonlinear response [9136-44] V. A. Trofimov, I. E. Kuchik, N. V. Levitskiy, Lomonosov Moscow State Univ. (Russian Federation) 
9136 1A Control of soliton pattern through continuous external injection [9136-45]

A. Niang, F. Amrani, M. Salhi, H. Leblond, Univ. d'Angers (France); A. Komarov, Univ. d'Angers (France) and Institute of Automation and Electrometry (Russian Federation); F. Sanchez, Univ. d'Angers (France)

9136 1B Instantaneous frequency measurement of microwave signals in optical range using "frequency-amplitude" conversion in the $\pi$-phase-shiffed fiber-Bragg grating [9136-46] O. G. Morozov, A. A. Talipov, M. R. Nurgazizov, P. E. Denisenko, A. A. Vasilets, Kazan National Research Technical Univ. (Russian Federation)

9136 1C Engaging new dimensions in nonlinear optical spectroscopy using auxiliary beams of light [9136-47]

J. S. Ford, D. S. Bradshaw, D. L. Andrews, Univ. of East Anglia (United Kingdom)

9136 IF Nonlinear picosecond pulse transformation in large-core microstructured fibers [9136-50] A. S. Pasishnik, S.I. Vavilov State Optical Institute (Russian Federation); S. O. Leonov, Bauman Moscow State Technical Univ. (Russian Federation)

913611 Investigation of the separate optical nonlinear contributions of the core and cladding materials of silicon photonics slotted waveguides [9136-53]

W. Zhang, Institut d'Électronique Fondamentale, Univ. Paris-Sud, CNRS (France); S. Serna, Institut d'Électronique Fondamentale, Univ. Paris-Sud, CNRS (France) and Lab. Charles Fabry, Institut d'Optique (France); N. Dubreuil, Lab. Charles Fabry, Institut d'Optique (France); E. Cassan, Institut d'Électronique Fondamentale, Univ. Paris-Sud, CNRS (France)

$91361 \mathrm{~J}$ Linear electro-optical scattering from ferroelectric nanocrystals [9136-54] D. T. Trinh, Lab. de Photonique Quantique et Moléculaire, CNRS (France), Institut D'Alembert, Ecole Normale Supérieure de Cachan (France), and Hanoi National Univ. of Education (Viet Nam); V. Shynkar, J. Zyss, Lab. de Photonique Quantique et Moléculaire, CNRS (France) and Institut D'Alembert, Ecole Normale Supérieure de Cachan (France)

9136 1K Effect factors of temperature measurements by femtosecond time-resolved CARS [9136-56] Y. Zhao, S. Zhang, Z. Zhang, Z. Dong, D. Chen, Z. Zhang, Y. Xia, Harbin Institute of Technology (China)

9136 1L Proposal of ultra-compact NAND/NOR/XNOR all-optical logic gates based on a nonlinear $3 \times 1$ multimode interference [9136-57]

M. Tajaldini, M. Z. Mat Jafri, Univ. Sains Malaysia (Malaysia)

$91361 \mathrm{M}$ Influence of photonic crystal fiber manufacturing inaccuracies on supercontinuum generation [9136-58]

M. Napierala, Z. Holdynski, M. Szymanski, M. Murawski, Military Univ. of Technology (Poland) and InPhoTech Ltd. (Poland); P. Mergo, Univ. of Maria Curie-Skłodowska (Poland); P. Marc, L. R. Jaroszewicz, Military Univ. of Technology (Poland); T. Nasilowski, Military Univ. of Technology (Poland) and InPhoTech Ltd. (Poland)

9136 IN Experimental demonstration of all optical XOR and XNOR gates for differential phase modulated data [9136-59]

R. Kakarla, D. Venkitesh, Indian Institute of Technology Madras (India) 
913610 Cross-absorption as a limit to heralded silicon photon pair sources [9136-60]

C. A. Husko, A. S. Clark, M. J. Collins, The Univ. of Sydney (Australia); A. De Rossi, S. Combrié, G. Lehoucq, Thales Research and Technology (France); I. H. Rey, Univ. of St. Andrews (United Kingdom); T. F. Krauss, Univ. of St. Andrews (United Kingdom) and The Univ. of York (United Kingdom); C. Xiong, B. J. Eggleton, The Univ. of Sydney (Australia)

9136 1P NLSE-based model of a random distributed feedback fiber laser [9136-61] S. V. Smirnov, Novosibirsk State Univ. (Russian Federation) and Aston Univ. (United Kingdom); D. V. Churkin, Novosibirsk State Univ. (Russian Federation) and Aston Univ. (United Kingdom) and Institute of Automation and Electrometry (Russian Federation)

9136 IR Multipolar nonlinear light-matter interactions with Gaussian vector beams [9136-63] M. J. Huttunen, Tampere Univ. of Technology (Finland) and Aalto Univ. (Finland); J. Mäkitalo, G. Bautista, M. Kauranen, Tampere Univ. of Technology (Finland)

9136 is Delay-based reservoir computing using semiconductor ring lasers [9136-64] R. M. Nguimdo, G. Verschaffelt, J. Danckaert, G. Van der Sande, Vrije Univ. Brussel (Belgium)

9136 1T Spatial extreme events in a photorefractive single-feedback system [9136-65] N. Marsal, V. Caullet, D. Wolfersberger, M. Sciamanna, Supélec (France) and Lab. Matériaux Optiques, Photonique et Systèmes (France)

9136 IV Modeling Kerr frequency combs using the Lugiato-Lefever equation: a characterization of the multistable landscape [9136-67]

P. Parra-Rivas, Vrije Univ. Brussel (Belgium) and Instituto de Fisica Interdisciplinar y Sistemas Complejos (Spain); D. Gomila, M. A. Matias, Instituto de Fisica Interdisciplinar y Sistemas Complejos (Spain); F. Leo, Univ. Gent (Belgium) and IMEC (Belgium); S. Coen, The Univ. of Auckland (New Zealand); L. Gelens, Vrije Univ. Brussel (Belgium) and Stanford Univ. School of Medicine (United States)

9136 1X Synthetic diamond as a new material for on-chip nonlinear wavelength converters [9136-69]

N. Vermeulen, Vrije Univ. Brussel (Belgium); J. E. Sipe, Univ. of Toronto (Canada); L. G. Helt, Macquarie Univ. (Australia); H. Thienpont, Vrije Univ. Brussel (Belgium)

$91361 Y$ Self-action effects in semiconductor quantum dots [9136-70]

V. S. Dneprovskii, A. R. Kanev, M. V. Kozlova, A. M. Smirnov, Lomonosov Moscow State Univ. (Russian Federation)

913620 Electrical nonlinear response of a photomixer for applications in ultrafast measurements [9136-73]

F. L. Constantin, Lab. PhLAM, CNRS (France) 


\title{
Part B Quantum Optics
}

\author{
QUANTUM OPTICS I
}

$913624 \quad$ High performing SPS based on native NIR-emitting single colour centers in diamond (Invited Paper) [9136-80]

D. Gatto Monticone, Univ. degli Studi di Torino (Italy), Istituto Nazionale di Fisica Nucleare (Italy), and Consorzio Nazionale Interuniversitario per le Scienze Fisiche della Materia (Italy); P. Traina, Istituto Nazionale di Ricerca Metrologica (Italy); E. Moreva, Istituto Nazionale di Ricerca Metrologica (Italy) and Lomonosov Moscow State Univ. (Russian Federation); J. Forneris, Univ. degli Studi di Torino (Italy), Istituto Nazionale di Fisica Nucleare (Italy), and Consorzio Nazionale Interuniversitario per le Scienze Fisiche della Materia (Italy); M. Levi, Univ. degli Studi di Torino (Italy); G. Brida, I. P. Degiovanni, G. Amato, L. Boarino, Istituto Nazionale di Ricerca Metrologica (Italy); P. Olivero, Univ. degli Studi di Torino (Italy), Istituto Nazionale di Fisica Nucleare (Italy), and Consorzio Nazionale Interuniversitario per le Scienze Fisiche della Materia (Italy); M. Genovese, Istituto Nazionale di Ricerca Metrologica (Italy)

913627 Optimized QKD BB84 protocol using quantum dense coding and CNOT gates: feasibility based on probabilistic optical devices [9136-83]

A. Gueddana, M. Attia, R. Chatta, SUP'COM (Tunisia)

913628 The study of reducing the effect of detector saturation on ghost imaging [9136-84]

R. Q. He, Q. Chen, W. W. Zhang, J. Shu, Nanjing Univ. of Science and Technology (China)

\section{QUANTUM OPTICS II}

$91362 \mathrm{~B}$ Position-dependent photon operators in the quantization of the electromagnetic field in dielectrics at local thermal equilibrium [9136-87]

M. Partanen, T. Häyrynen, J. Oksanen, J. Tulkki, Aalto Univ. (Finland)

$91362 \mathrm{C}$ Optical four-wave mixing and generation of squeezed light in an optomechanical cavity driven by a bichromatic field [9136-88]

R. Garcés, G. J. de Valcárcel, Univ. de València (Spain)

9136 2D Quantum information with optical photons in hybrid molecule-superconducting qubit system [9136-89]

S. Das, Niels Bohr Institute (Denmark); S. Faez, Leiden Institute of Physics (Netherlands);

A. S. Sørensen, Niels Bohr Institute (Denmark)

\section{QUANTUM OPTICS III: MANIPULATION OF Q STATES OF LIGHT}

$91362 \mathrm{E} \quad$ Recovery of qubit coherence by noise-eater technique (Invited Paper) [9136-90]

M. Gavenda, L. Čelechovská, M. Dušek, R. Filip, Palacký Univ. Olomouc (Czech Republic)

$91362 \mathrm{G}$ Slow light in evanescently coupled optical cavities containing quantum dots (Best Student Paper Award) [9136-92]

E. Ergecen, Middle East Technical Univ. (Turkey) 
$91362 \mathrm{H} \quad$ Generation of correlated photon pairs in micro/nano-fibers [9136-93]

X. Li, L. Cui, C. Guo, Tianjin Univ. (China); Y. H. Li, Z. Y. Xu, L. J. Wang, Tsinghua Univ. (China);

W. Fang, Zhejiang Univ. (China)

\section{QUANTUM OPTICS IV: APPLICATIONS}

9136 2K Nanometric surface probing through ultra-cold atoms [9136-98]

M. Ali Khan, European Lab. for Non-Linear Spectroscopy (Italy) and Karlsruhe School of Optics (Germany); F. Schaefer, Kyoto Univ. (Japan); W. H. P. Pernice, Karlsruhe School of Optics (Germany); F. S. Cataliotti, European Lab. for Non-Linear Spectroscopy (Italy)

\section{POSTER SESSION}

913620 Nearly deterministic loading of a single cesium atom in a magneto-optical trap and in a microscopic optical tweezer by feedback control [9136-100]

B. Liu, J.-M. Wang, W.-T. Diao, J.-Y. Wang, G. Jin, J. He, Shanxi Univ. (China)

Author Index 


\title{
Conference Committee
}

\author{
Symposium Chairs
}

Francis Berghmans, Vrije Universiteit Brussel (Belgium)

Ronan Burgess, European Commission (Belgium)

Jürgen Popp, Institut für Photonische Technologien e.V. (Germany)

Peter Hartmann, SCHOTT AG (Germany)

Honorary Symposium Chair

Hugo Thienpont, Vrije Universiteit Brussel (Belgium)

\section{Part A Nonlinear Optics and its Applications}

Conference Chairs

Benjamin J. Eggleton, The University of Sydney (Australia)

Alexander L. Gaeta, Cornell University (United States)

Neil G. R. Broderick, The University of Auckland (New Zealand)

Conference Programme Committee

Stephane Coen, The University of Auckland (New Zealand)

Arnaud Couairon, Ecole Polytechnique (France)

Richard M. De La Rue, University of Malaya (Malaysia) and University of Glasgow (United Kingdom)

Christophe Dorrer, University of Rochester (United States)

Majid Ebrahim-Zadeh, ICFO - Institut de Ciències Fotòniques (Spain)

Goëry Genty, Tampere University of Technology (Finland)

John D. Harvey, The University of Auckland (New Zealand)

Peter Horak, University of Southampton (United Kingdom)

Colin J. McKinstrie, Alcatel-Lucent Bell Laboratories (United States)

Dragomir N. Neshev, The Australian National University (Australia)

Leif Katsuo Oxenløwe, Technical University of Denmark (Denmark)

Siddharth Ramachandran, Boston University (United States)

John E. Sipe, University of Toronto (Canada)

\section{Session Chairs}

Nonlinear Quantum Optics

Benjamin J. Eggleton, CUDOS (Australia) 
High Power Effects

KaFai Mak, Max-Planck-Institut für die Physik des Lichts (Germany)

Nanophotonics

Alexander L. Gaeta, Cornell University (United States)

Cavity Solitons

Marc Hälterman, Université Libre de Bruxelles (Belgium)

Specialty Fibres

John M. Dudley, Université de Franche-Comté (France)

Applications of Nonlinear Optics

Miro Erkintalo, Tampere University of Technology (Finland)

Fibre Lasers I

Neil G. R. Broderick, The University of Auckland (New Zealand)

Fibre Lasers II

Frank W. Wise, Cornell University (United States)

Parametric Effects

Frank W. Wise, Cornell University (United States)

\section{Part B Quantum Optics}

\section{Conference Chairs}

Alexander V. Sergienko, Boston University (United States)

Arno Rauschenbeutel, Vienna Center for Quantum Science and Technology (Austria)

Thomas Durt, Ecole Centrale Marseille (France)

\section{Conference Programme Committee}

Alain Aspect, Institut d'Optique Graduate School (France)

Victor Balykin, Institute of Spectroscopy (Russian Federation)

Vladimir Buzek, Slovak Academy of Sciences (Slovakia)

Berthold-Georg Englert, National University of Singapore (Singapore)

Serge Massar, Université Libre de Bruxelles (Belgium)

Gerard J. Milburn, The University of Queensland (Australia)

Jeremy L. O'Brien, University of Bristol (United Kingdom)

Paolo Tombesi, Universitá degli Studi di Camerino (Italy)

Vlatko Vedral, University of Oxford (United Kingdom)

Victor N. Zadkov, Lomonosov Moscow State University

(Russian Federation) 
Anton Zeilinger, Universität Wien (Austria)

\section{Session Chairs}

Quantum Optics I

Thomas Durt, Ecole Centrale Marseille (France)

Quantum Optics II

Sara Ducci, Université Paris 7-Denis Dideroł (France)

Quantum Optics III: Manipulation of Q States of Light

Paolo Traina, Istituto Nazionale di Ricerca Metrologica (Italy)

Quantum Optics IV: Applications

Thomas Durt, Ecole Centrale Marseille (France) 
Proc. of SPIE Vol. 9136 913601-14

Downloaded From: https://www.spiedigitallibrary.org/conference-proceedings-of-spie on 26 Apr 2023 Terms of Use: https://www.spiedigitallibrary.org/terms-of-use 


\section{Introduction}

This conference on Quantum Optics in the frame of the SPIE Photonics Europe 2014 was arranged for the third time in Brussels, Belgium. It was devoted to the recent scientific advances at the interface between quantum optics and atom optics. During the last decades, the studies of fundamental issues in quantum mechanics exploded from their originally confidential circle of specialists and became a major field of research that covers a large range of sub-disciplines, from nanotechnologies to quantum optics, including Bose-Einstein condensates, single photon sources, squeezed light, technologies for engineering quantum states and manipulating single atoms and ions, as well as quantum dots, etc. Although those researches are clearly foundationally oriented, they fit closely to experiments and already found amazingly successful applications in top-level metrology (spectroscopy, atomic clocks, measure of fundamental constants and so on) and quantum information (quantum cryptography and computing). The conference aimed at bringing a great opportunity to listen to some of the world renowned experts in these interconnected disciplines, as well as to discover new trends that result from the convergence of these fields. It offered an updated review of recent activities both in theoretical and experimental research. The conference programme and all manuscripts included in this volume cover the following topics:

- Nonclassical field states

- Quantum entanglement and EPR states

- Quantum states engineering and reconstruction

- Quantum memory for light, quantum interfaces, slow light, EIT

- Optomechanical devices

- Quantum communication and information processing

- Colour centers in diamond

- Quantum cryptography

- Nonlinear optics in graphene

- Quantum light in cavity

- Quantum key distribution

In conclusion, we thank all the conference participants and the authors of the submitted manuscripts for their contributions. Our special thanks also go the members of the program committee of this conference for their involvement into setting a good program.

Thomas Durt Alexander V. Sergienko 
Proc. of SPIE Vol. 9136 913601-16

Downloaded From: https://www.spiedigitallibrary.org/conference-proceedings-of-spie on 26 Apr 2023 Terms of Use: https://www.spiedigitallibrary.org/terms-of-use 